\title{
A Compact LTCC-Based Ku-Band Transmitter Module
}

\author{
Chang-Ho Lee, Member, IEEE, Albert Sutono, Sangwoo Han, Kyutae Lim, Member, IEEE, Stéphane Pinel, \\ Emmanouil M. Tentzeris, Member, IEEE, and Joy Laskar, Member, IEEE
}

\begin{abstract}
In this paper, we present design, implementation, and measurement of a three-dimensional (3-D)-deployed RF front-end system-on-package (SOP) in a standard multi-layer low temperature co-fired ceramic (LTCC) technology. A compact 14 GHz GaAs MESFET-based transmitter module integrated with an embedded band pass filter was built on LTCC 951AT tapes. The up-converter MMIC integrated with a voltage controlled oscillator (VCO) exhibits a measured up-conversion gain of $15 \mathrm{~dB}$ and an IIP3 of 15 $\mathrm{dBm}$, while the power amplifier (PA) MMIC shows a measured gain of $31 \mathrm{~dB}$ and a 1-dB compression output power of $26 \mathrm{dBm}$ at $14 \mathrm{GHz}$. Both MMICs were integrated on a compact LTCC module where an embedded front-end band pass filter (BPF) with a measured insertion loss of $3 \mathrm{~dB}$ at $14.25 \mathrm{GHz}$ was integrated. The transmitter module is compact in size $\left(400 \times 310 \times 35.2 \mathrm{mil}^{3}\right)$, however it demonstrated an overall up-conversion gain of $41 \mathrm{~dB}$, and available data rate of $32 \mathrm{Mbps}$ with adjacent channel power ratio (ACPR) of $42 \mathrm{~dB}$. These results suggest the feasibility of building highly SOP integrated RF front ends for microwave and millimeter wave applications.
\end{abstract}

Index Terms-ACPR, coupled line filter, DBS, dual gate mixer, Ku-band, LTCC, MESFET, MMIC, negative resistance VCO, power amplifier, satellite communications, SOP, transmitter, up-converter.

\section{INTRODUCTION}

$\mathbf{T}$ O SATISFY the significant worldwide demand for higher data rates and broadband transmission, applications of satellite communication systems in the $\mathrm{Ku} / \mathrm{Ka}$-band range are expanding due to their large available bandwidth [1]. However, there is still a deficiency in economical high-frequency components for low-cost transceivers. The current drawbacks of most commercially available microwave and millimeter wave front-ends, such as the Ku-band satellite transceivers for outdoor units, are their relatively large size and heavy weight which are primarily caused by discrete components such as filters, and separately located module. The implementation of a compact module is the key issue for reduction in cost, size and system complexity. Multilayer ceramic and organic-based SOP implementation are capable of overcoming these limitations by integrating components as part of the module package that would have otherwise been acquired in

Manuscript received March 20, 2002; revised June 10, 2002. This work was supported by the NSF Packaging Research Center, Georgia Institute of Technology, Yamacraw Design Center, and the NSF Award.

C.-H. Lee and S. Han are with RF Solutions, Inc., Norcross, GA 30071 USA. A. Sutono is with Infinera, Inc., Sunnyvale, CA 94089 USA.

K. Lim, S. Pinel, E. M. Tentzeris, and J. Laskar are with the Packaging Research Center, Yamacraw Design Center, School of Electrical and Computer Engineering, Georgia Institute of Technology, Atlanta, GA 30332 USA.

Digital Object Identifier 10.1109/TADVP.2002.805315 discrete form. On-package components not only miniaturize the module, but also minimize or eliminate the need for the discrete components and thereby reduce the assembly time and cost as well. LTCC-based modules demonstrated so far [2]-[4] were dedicated for phase-array applications. The feasibility of implementing a LTCC integrated filter has been demonstrated in [5], [6] for L-band application. The same concept is now extended for Ku-band applications. This paper demonstrates a development of a compact functional $14 \mathrm{GHz}$ LTCC-based transmitter module suitable for outdoor transmitter units. The module features an integrated stripline filter and MMIC chipsets fabricated in a commercial Triquint's GaAs MESFET processes. Experimental results demonstrate that this transmitter module is suitable for the satellite out door units (ODUs) with data rate up to $32 \mathrm{Mbps}$ and an ACPR of $42 \mathrm{~dB}$.

\section{System DESIGN CONSIDERATION}

In the design of the transmitter module, the main concern is to meet transmit power level and linearity as well as to filter unwanted spurious signals. The system was designed in modular form, consisting of two MMIC chips, an up-converter MMIC and a power amplifier MMIC, and a coupled strip line BPF. Two MMIC chips are mounted and wire-bonded on the LTCC substrate incorporating with an embedded coupled strip line BPF. This configuration was selected to apply the filtering network between two MMICs and to avoid the thermal effect on the up-converter MMIC. The LTCC is composed of 20 layers of Dupont 951 fired ceramic, fabricated by the NSC Corporation. However, only 10 layers are part of this transmitter module. Each layer of the structure is 3.7 mil thick.

The schematic of the implemented double conversion transmitter module is shown in Fig. 1. The up-converter MMIC consists of an IF amplifier, a dual-gate mixer, a low phase noise VCO, a local oscillator (LO) buffer amplifier and a RF amplifier as shown in Fig. 2. The PA MMIC consists of a five-stage driver amplifier and a one-stage power amplifier as shown in Fig. 3. The gain requirement of the up-converter was determined by the IF power available from the IF amplifier and the available LO power. The required output power of the up-converter was determined while overcoming the losses in the BPF and bond wires between MMICs and BPF, which was required to put the driver amplifier and power amplifier in compression.

\section{MMic Design And Measured Performance}

Two MMICs are fabricated using the commercial $0.6 \mu \mathrm{m}$ GaAs MESFET process at Triquint Semiconductor. The entire 


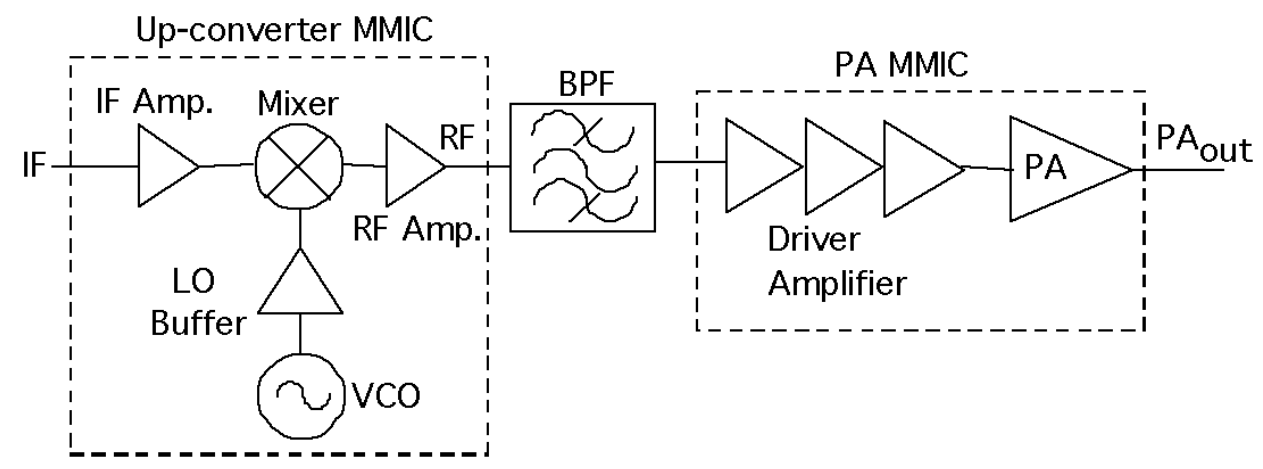

Fig. 1. Block diagram of double conversion transmitter.

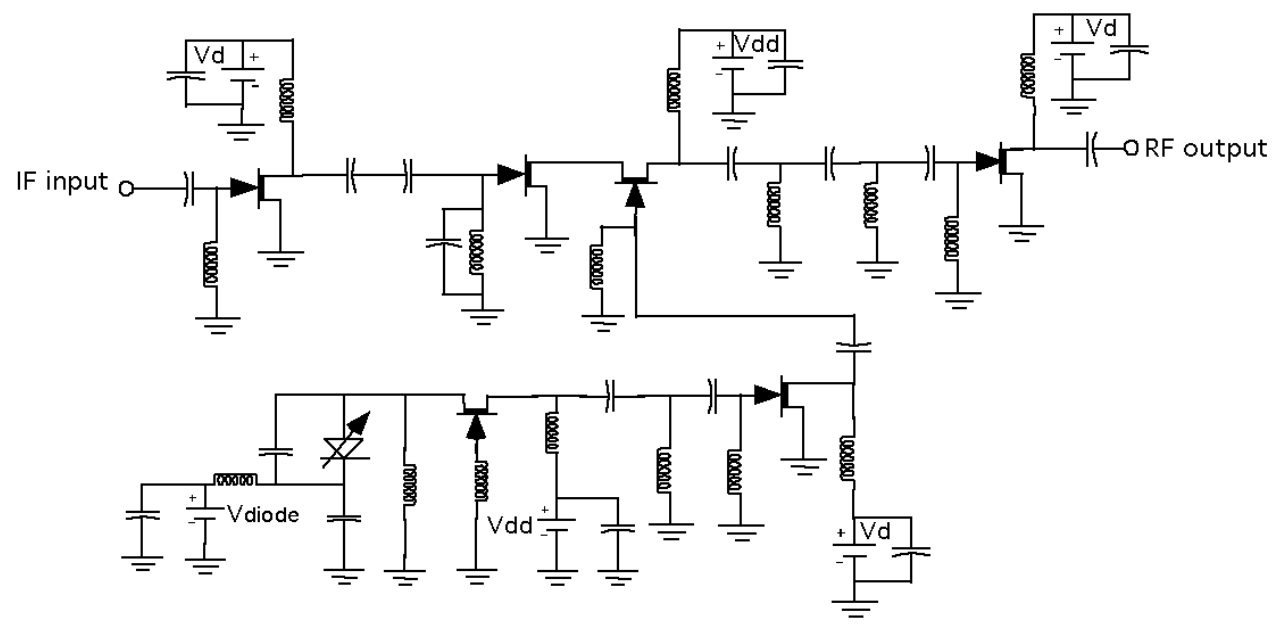

Fig. 2. Schematic diagram of the up-converter MMIC.

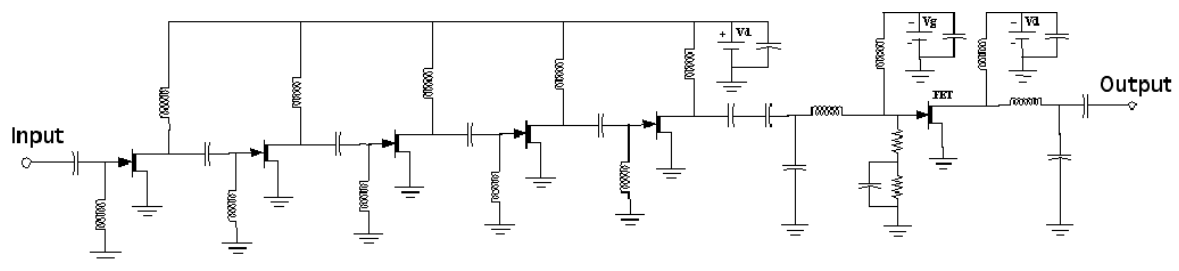

Fig. 3. Schematic diagram of the PA MMIC.

transmitter chain exhibits a total conversion gain of $41 \mathrm{~dB}$ and an output power of $26 \mathrm{dBm}$ incorporating the losses and mismatches caused by the bond wires and the filter over a $500 \mathrm{MHz}$ RF bandwidth.

\section{A. Up-Converter MMIC}

The dual gate topology provides simplicity, lower distortion, and good LO-IF isolation. Having conversion gain eliminates the need for additional gain stages to compensate for the losses. In this design, good LO to RF isolation and image signal rejection has been achieved through the use of a LTCC BPF at the $\mathrm{RF}$ port instead of using balanced mixer topology. The conversion gain and IIP 3 at the output RF frequency $(14 \mathrm{GHz})$ are $2 \mathrm{~dB}$ and $15 \mathrm{dBm}$, respectively, at a LO power of $14 \mathrm{dBm}$, as shown in Fig. 4(a). Fig. 4(b) shows a LO to RF isolation of $-10 \mathrm{~dB}$ at an $\mathrm{LO}$ frequency from $12 \mathrm{GHz}$ to $14 \mathrm{GHz}$. To reject the $\mathrm{LO}$ feed-through signal at the output, the BPF was inserted between the RF amplifier and the driver amplifier.
The IF amplifier was designed to increase the gain of the dual gate mixer and to produce low noise figure of the entire up-converter, as well as to provide better input matching to the IF port of the mixer. Reactive matching was used to produce good noise figure while sacrificing the broadband input match. Experimental data shows the more than $10 \mathrm{~dB}$ gain, 3 to $4 \mathrm{~dB}$ noise figure (NF) and IIP3 of $5 \mathrm{dBm}$ between $600 \mathrm{MHz}$ and 1200 MHz. It also shows a wide band output match (S22 <-10 dB) that can provide wide matching with the IF port of the dual gate mixer. Fig. 5 shows the measured gain and return loss performance from 0.1 to $5 \mathrm{GHz}$.

The output power of the up-converter has to be sufficient to overcome the loss of a bandpass filter while maintaining the desired output power of the entire module. The RF amplifier with a measured gain of $5 \mathrm{~dB}$ and 1-dB compression point of $7 \mathrm{dBm}$ was implemented to increase the conversion gain of the entire up-converter MMIC, and provides a good output matching with the BPF network. The RF amplifier uses 


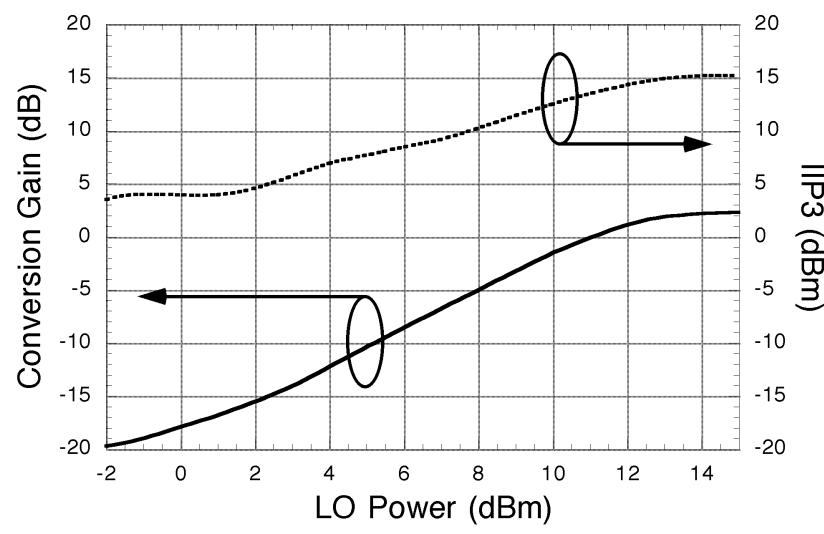

(a)

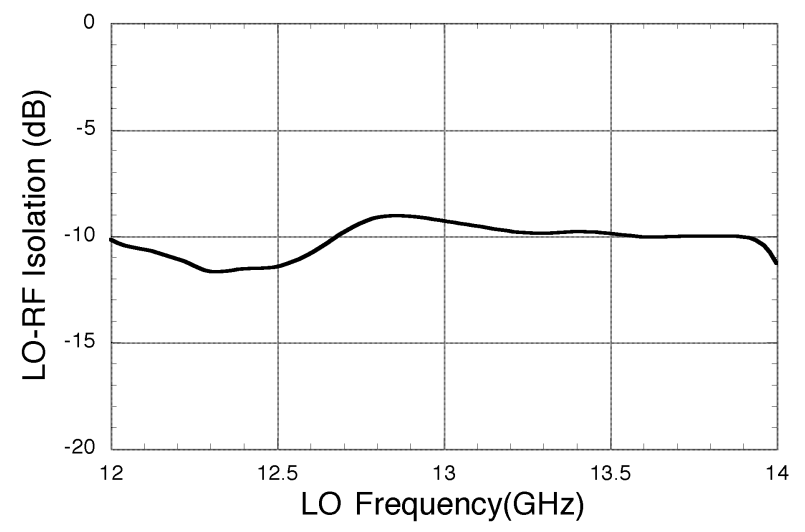

(b)

Fig. 4. (a) Measured conversion gain and IIP3 versus LO power for the mixer. (b) Measured LO-to-RF isolation versus LO frequency for the mixer.

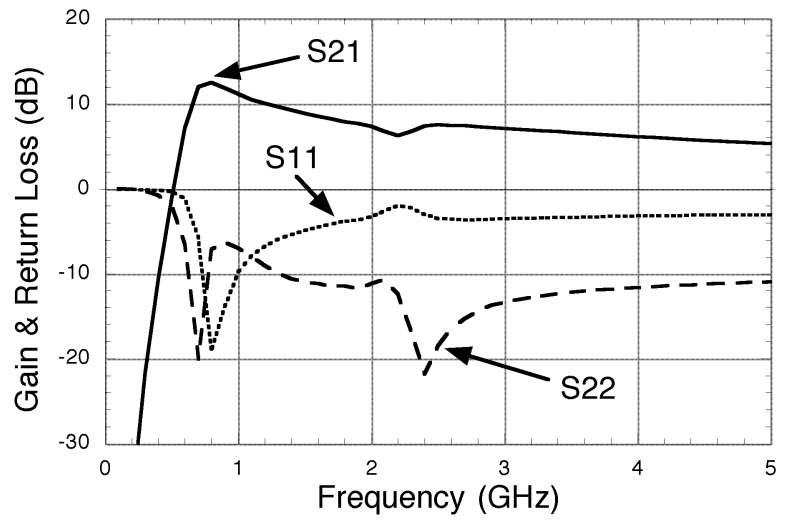

Fig. 5. Measured gain and return loss of the IF amplifier.

a single-stage common-source amplifier topology incorporating reactive matching. The RF amplifier is used to boost the upper side band (USB) output power and also contains a high pass filter network, in its input matching network, to reduce the level of IF signal from the dual gate mixer. Fig. 6 shows the measured gain and return loss performance from 10 to $18 \mathrm{GHz}$.

The LO signal is generated by an integrated local oscillator and buffer amplifier. This maintains stability of the spurious oscillations over required frequency band. The VCO design utilizes a negative resistance common-gate configuration to

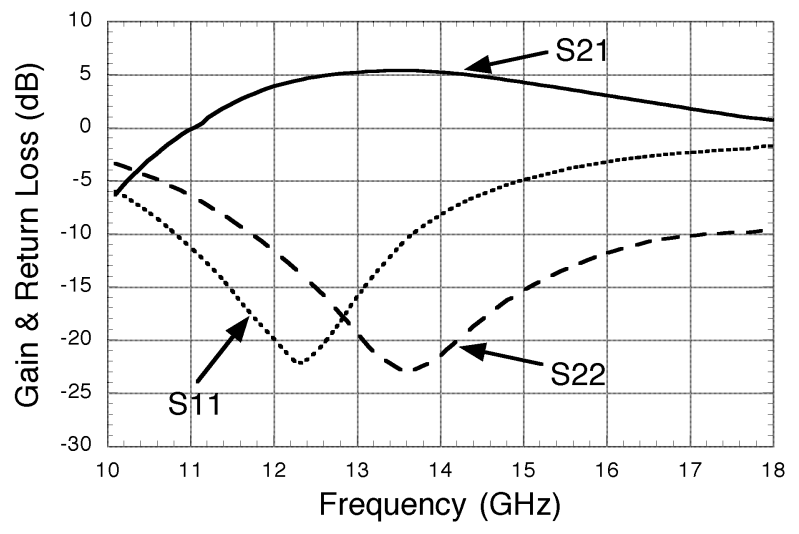

Fig. 6. Measured gain and return loss of the RF amplifier.

generate strong negative resistance by inductive feedback and to facilitate varactor diode tuning and implementation. In this design, the device size is optimized for lower NF in the frequency range. The load impedance is carefully designed to increase the $Q$-factor of the resonator. Although a much wider tuning bandwidth can be obtained by placing the varactor on the gate path, the varactor is incorporated in the resonator on the source path to reduce its noise contribution to the VCO [7]. To eliminate undesirable low frequency oscillations, a LC network is also incorporated as a high pass filter on the output path. Fig. 7(a) shows the measured signal spectrum of the $\mathrm{VCO}$ over a $5 \mathrm{MHz}$ span showing a phase noise of -110 $\mathrm{dBc} / \mathrm{Hz}$ at a $1 \mathrm{MHz}$ offset. An excellent measurement of the second harmonic suppression of $40 \mathrm{~dB}$ or more was observed across the entire power and frequency range, with no parasitic oscillations detected, as shown in Fig. 7(b). Fig. 8 shows the measured frequency and output power level as a function of the varactor voltage. A frequency tuning range of $500 \mathrm{MHz}$, ranging from $11.8 \mathrm{GHz}$ to $13.3 \mathrm{GHz}$, with uniform phase noise performance was achieved over a tuning voltage range of -1 to 3 volts. In addition to desensitizing the VCO to the external load impedance, the LO buffer amplifier was designed to deliver the required drive power $(14 \mathrm{dBm})$ to the mixer and to provide a good match at the LO port of the mixer. It is essentially responsible to provide an unconditionally stable operation for the up-converter, both in the presence and absence of a LO signal. The LO buffer amplifier provides a measured gain of 7 $\mathrm{dB}$ at $13 \mathrm{GHz}$, and has an input 1-dB compression point of 18 $\mathrm{dBm}$. The measured gain and return loss performance is shown in Fig. 9.

\section{B. PA MMIC}

The driver amplifier specification was derived to maximize the performance of the entire transmitter chain. The output power of the driver amplifier had to be sufficient enough to overcome the loss of a BPF and to drive the power amplifier into saturation from 14 to $14.5 \mathrm{GHz}$. This output power level was determined to be more than $24 \mathrm{dBm}$. The required gain for the driver amplifier was determined from the output power of the up-converter and the associated losses of the filter and the bonding wire between them. The anticipated minimum power level available to the driver amplifier is about $-5 \mathrm{dBm}$. 


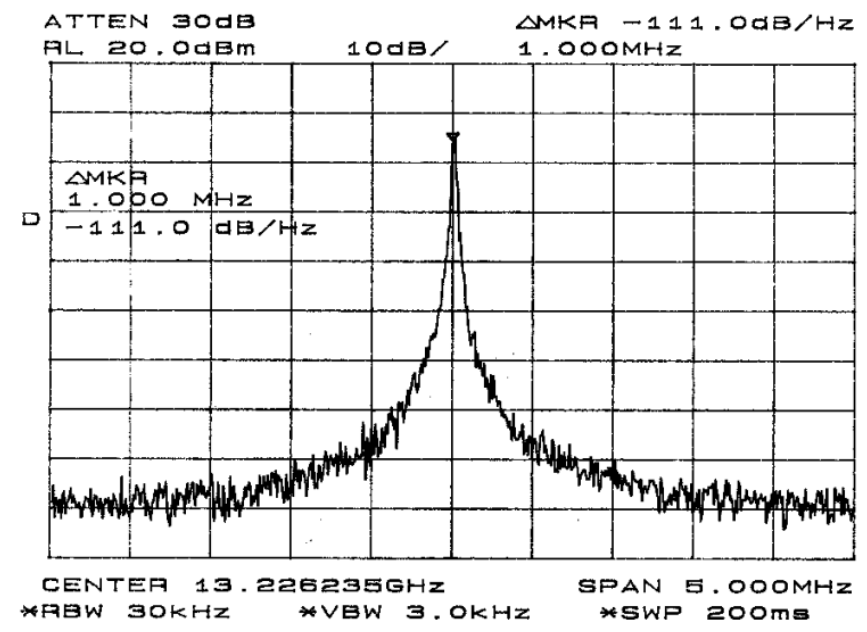

(a)

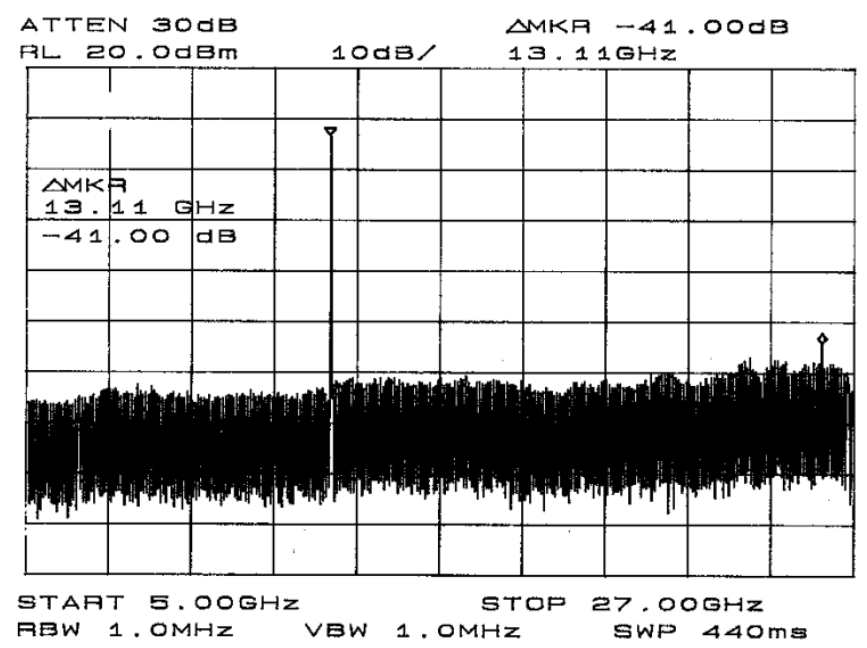

(b)

Fig. 7. (a) Measured output frequency spectrum of the VCO over a $5 \mathrm{MHz}$ span. (Phase noise: $-111 \mathrm{dBc} / \mathrm{Hz} @ 1 \mathrm{MHz}$ offset). (b) Measured harmonic performance for the VCO.

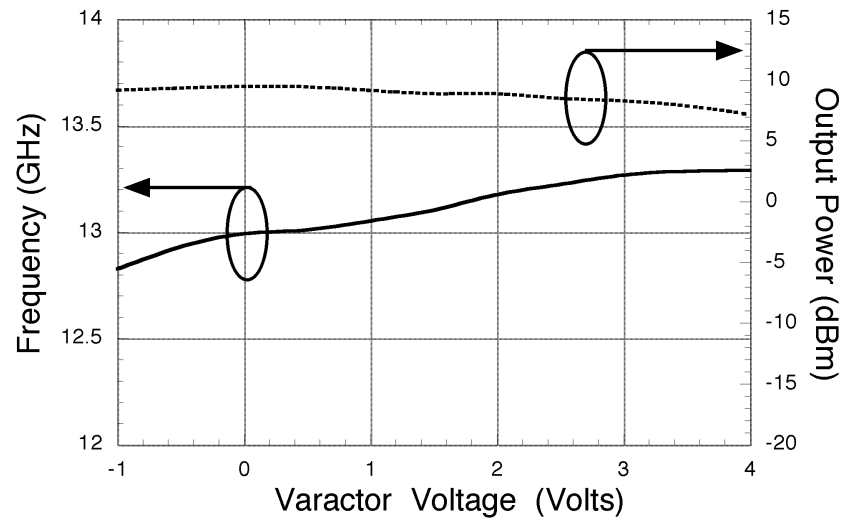

Fig. 8. Measured frequency and output power versus the varactor control voltage for the VCO.

Therefore, the driver amplifier required a minimum gain of $25 \mathrm{~dB}$ to produce the $20 \mathrm{dBm}$ of output power. In order to meet the specification and allow for process variations, the five-stage amplifier was designed to produce more than $26 \mathrm{~dB}$ of small signal gain from 14 to $14.5 \mathrm{GHz}$. The amplifier incorporated the

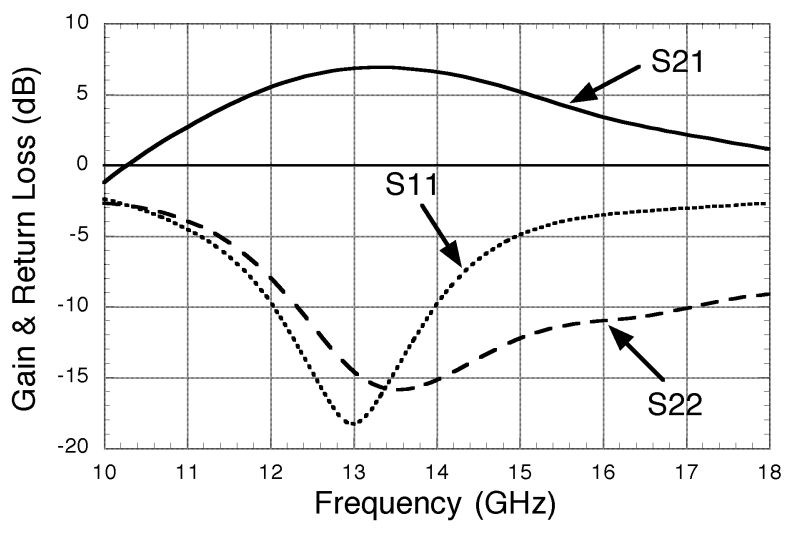

Fig. 9. Measured gain of return loss for the LO buffer amplifier.

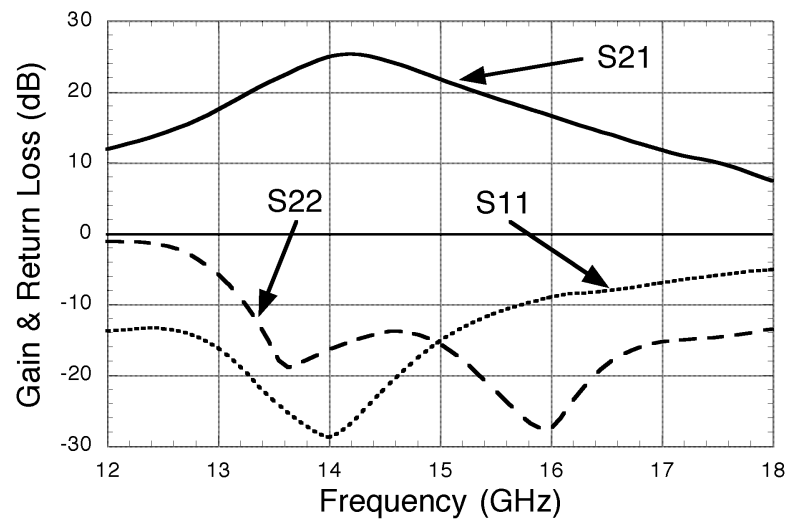

Fig. 10. Measured gain and return loss for the driver amplifier.

reactive matching as well as feedback resistance for unconditional stability. The resulting amplifier exhibited $26 \mathrm{~dB}$ of gain and return loss of better than $14 \mathrm{~dB}$ over the entire frequency band. When driven with $-5 \mathrm{dBm}$, the amplifier provides $21 \mathrm{dBm}$ of output power. The common-source topology and single bias supply scheme with self-biasing network is used to simplify the biasing circuitry. Fig. 10 shows the measured gain and return loss performance. The measurements show a 1-dB compression point of $22 \mathrm{dBm}$ across the required frequency range.

A class-A power amplifier was designed using a nonlinear large signal model (TOM3) and the dynamic load line method. To meet the design goals, a $1.5 \mathrm{~mm}$ device was selected for single-ended one-stage common source amplifier design. Lumped elements were used to realize the matching network. The PA exhibits about $6 \mathrm{~dB}$ of gain with a 1-dB compression point of $26 \mathrm{dBm}$ and a PAE of $30 \%$ as shown in Fig. 11. For an accurate inductor model, a commercial MoM simulator [8] is used and the distributed effects of metal interconnection in the layout were considered. This MMIC also includes pads for on-wafer testing and shunt capacitors on the bias pads to minimize parasitic effects of the dc probes.

\section{BAND Pass Filter Design AND MEASURED PERFORMANCE}

There are three configurations showing band pass filter response [9] that can be obtained from a pair of coupled strip lines 


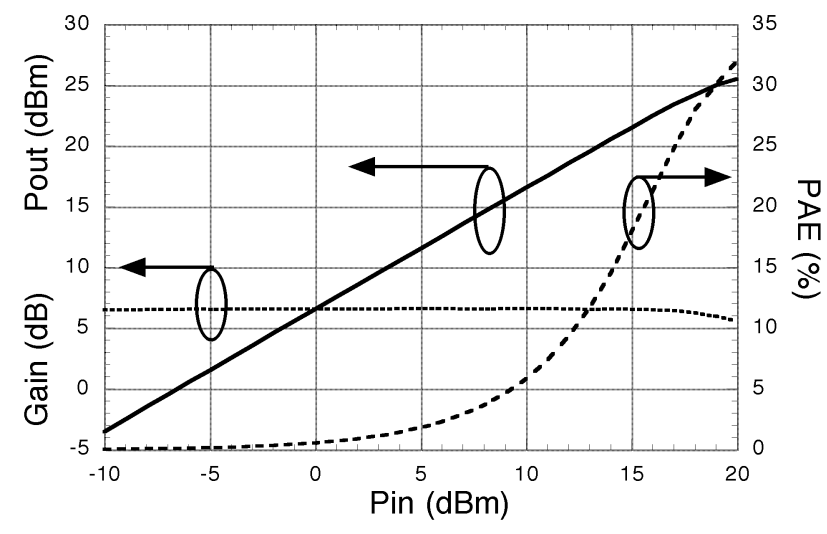

Fig. 11. Measured gain, output power, and PAE for the power amplifier.

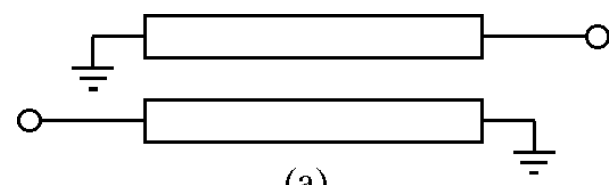

(a)

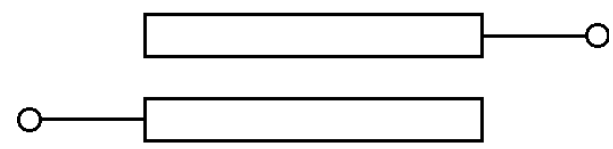

(b)

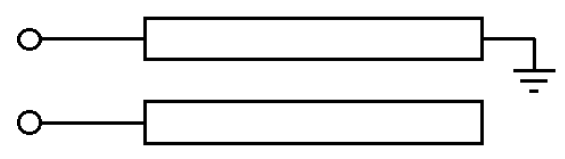

(c)

Fig. 12. Coupled line configuration.

by terminating two of the four ports in either open or short circuits, or by connecting the ends of the lines together. Fig. 12 shows single sections of the three possible coupled line BPFs. In most cases, it is necessary to cascade several segments of the filter in order to obtain the required performance such as bandwidth and insertion loss. Any number of segments can be cascaded when the input and output of a single segment are placed at the opposite side of the strips. But, in Fig. 12(c), only two segments can be cascaded because the input and output of a single segment are placed at the same end. In addition, it is easier to fabricate filter segments in form of open circuits rather than short circuits. Therefore, Fig. 12(b) is chosen for band pass filter implementation due to its inherent configurations. The image impedance of the coupled strip line filters differs from the characteristic impedance of an isolated strip [9]. Therefore, it is necessary to connect the filter strips with the coupled strips having different widths in order to reduce the loss resulting from mismatch at the terminals.

The BPF for the transmitter module was implemented in a coupled line filter topology on a multi-layer LTCC substrate to suppress the LO signal at $13 \mathrm{GHz}$ and image signal at $12 \mathrm{GHz}$ as well as suppress the harmonics and spurious signals. The

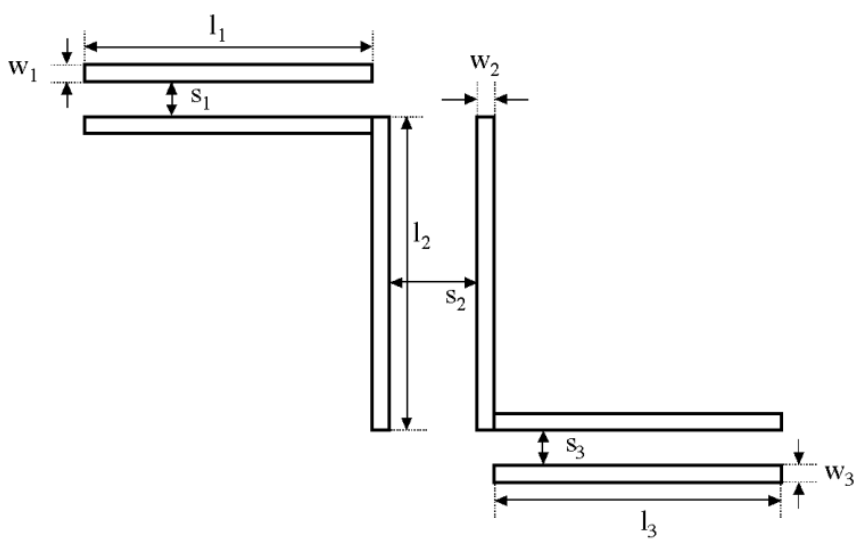

Fig. 13. Schematic diagram of folded edge-coupled strip line filter.

TABLE I

Summary of Measured Performance of the Fabricated LTCC COUPLED STRIP LINE FILTER

\begin{tabular}{|c|c|c|c|c|c|c|c|}
\hline & \multicolumn{2}{|c|}{ Dimension } & \multirow{2}{*}{$\begin{array}{c}\mathrm{f}_{\mathrm{c}} \\
(\mathrm{GHz})\end{array}$} & \multirow{2}{*}{$\begin{array}{l}\text { Insertion } \\
\text { loss (dB) }\end{array}$} & \multirow{2}{*}{$\begin{array}{c}\text { Return } \\
\text { loss (dB) }\end{array}$} & \multirow{2}{*}{$\begin{array}{c}\text { LO rejection } \\
\text { (dB) at } 13 \\
\text { GHz }\end{array}$} & \multirow{2}{*}{$\begin{array}{c}3 \mathrm{~dB} \\
\text { bandwidth } \\
(\mathrm{GHz})\end{array}$} \\
\hline & $1_{1}, l_{3}$ & $\mathrm{~S}_{2}$ & & & & & \\
\hline A & 66 & 16 & 14.527 & -3.27 & -29.61 & -19.71 & 1.1 \\
\hline B & 68 & 16 & 14.329 & -3.06 & -27.82 & -18.15 & 1.1 \\
\hline C & 69 & 16 & 14.229 & -3.14 & -21.91 & -17.05 & 1.1 \\
\hline D & 70 & 16 & 14.130 & -3.12 & -18.97 & -16.15 & 1.1 \\
\hline$E$ & 68 & 18 & 14.225 & -3.01 & -20.34 & -18.05 & 1 \\
\hline
\end{tabular}

number of coupled line segments depends on the filter order needed to meet the bandwidth specifications. Fig. 13 shows the schematic of the implemented LTCC-based three segment folded edge coupled line filter, where the middle segment was deployed perpendicular to the first and third segments for compactness. Eight filters were fabricated; all have the same dimensions for the $w_{1}, w_{2}, w_{3}$ and $s_{1}, s_{3}$ strips as well as $l_{2}$ strips, but have different dimensions for $l_{1}, l_{3}$ and $s_{2}$. Table I summarizes the comparison between the five filters, including the measured performance in terms of the center frequency $f_{c}$, insertion and return losses at $f_{c}$, LO rejection performance at $13 \mathrm{GHz}$, and 3 $\mathrm{dB}$ bandwidth. These filters were designed by incorporating a MoM electromagnetic (EM) simulator [8].

From the summary in Table I, it is obvious that the center frequency $f_{c}$ depends on $l_{I}, l_{3}$ by noting the variation for $f_{c}$ of $400 \mathrm{MHz}$ among the five filters. The role of $s$, spacing between coupled line affects the bandwidth and $Q$ of the filter. Also $w$, width of the coupled line, affects the center frequency. From the system point of view, Filter $\mathrm{E}$ is selected for module implementation because it meets well all the specifications. It exhibits 3 $\mathrm{dB}$ of insertion loss, $20 \mathrm{~dB}$ of return loss, and $0.2 \mathrm{~dB}$ of gain flatness between 13.9 to $14.4 \mathrm{GHz}$.

In Fig. 14, the measurement shows a slightly higher center frequency than simulation results. This can be explained by investigating the actual layout and implementation. In real implementation, via connecting the strip line to CPW is placed on the coupled line with 8 mil apart from the edge of strips because of the design rule for via process. This via location on strips reduced the actual length of the coupled strip line length by 5 to 10 mils. Therefore, the center frequency is expected to increase by the amount of the reduced strip line length. Meanwhile, because 


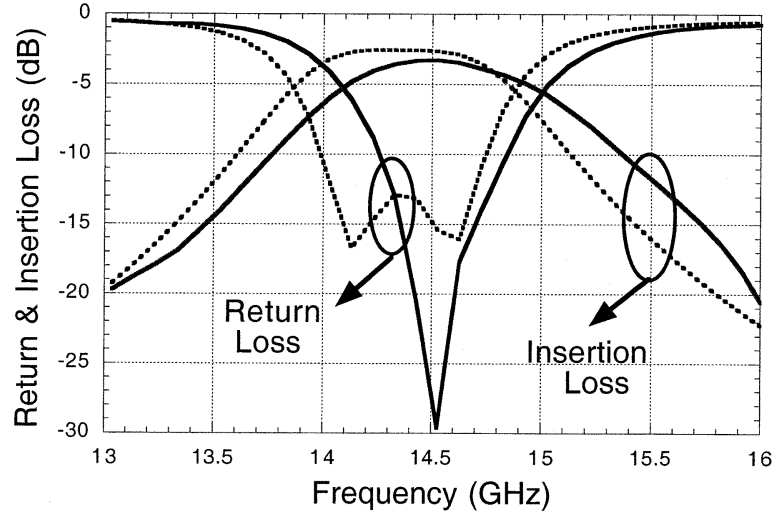

(a)

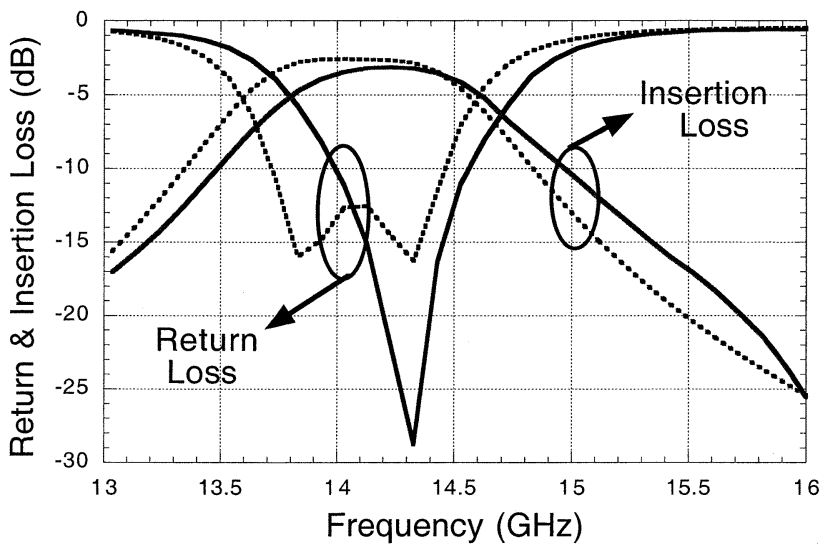

(c)

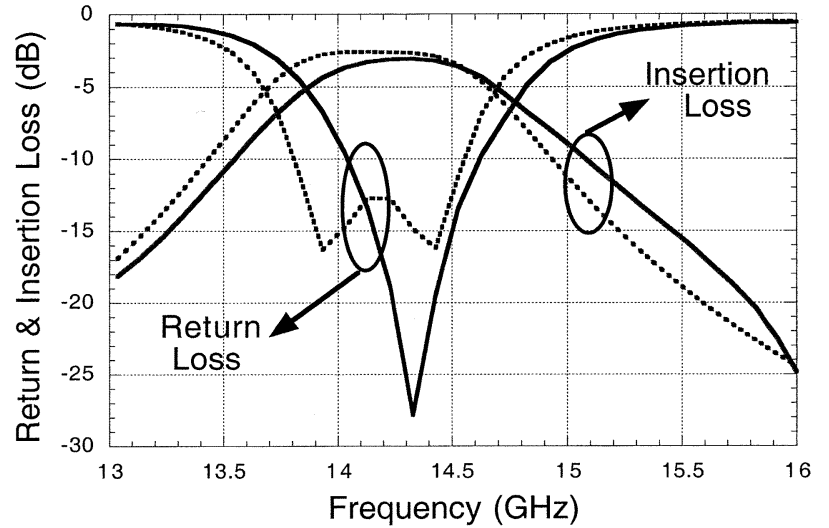

(b)

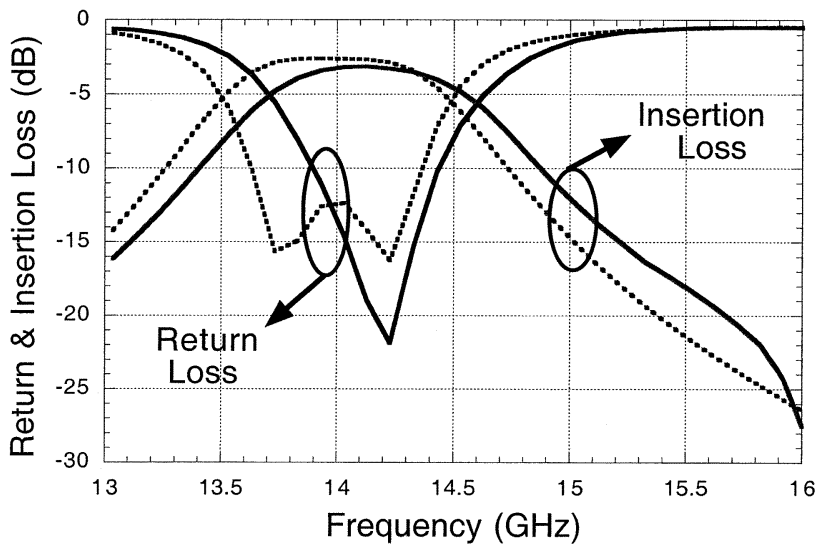

(d)

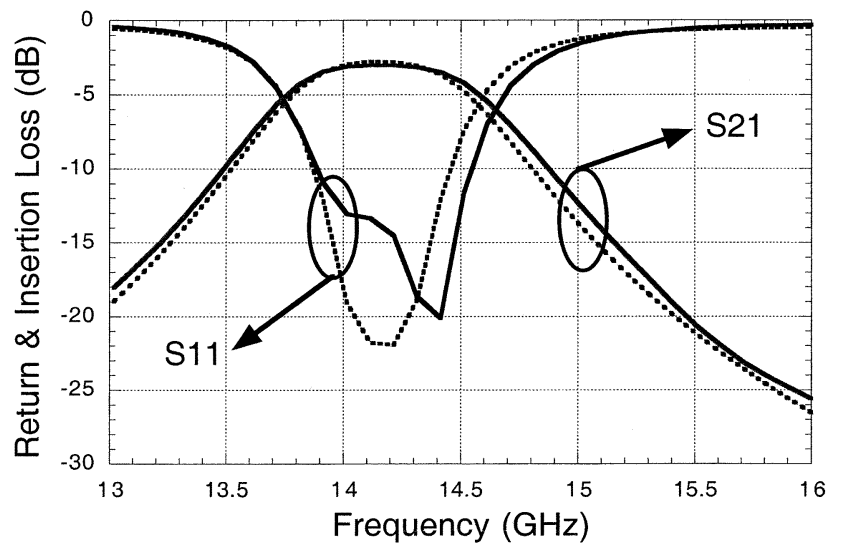

(e)

Fig. 14. Performance comparison of coupled strip line BPFs between measurement (solid line) and simulations (dashed line). (a) Filter A. (b) Filter B. (c) Filter C. (d) Filter D. (e) Filter E.

of its folded structure, additional coupling between two neighboring segments is expected and results in more parasitic capacitance between them. Therefore the additional coupling can compensate for the increasing effects on center frequency for some amount, which are overall slightly higher than the method of momentum (MoM) simulation results. Two reasons contribute to the discrepancy between measurement and simulation. First, the filter measurement includes the stripline to CPW transition that exhibits additional parasitic mechanisms. The simulation did not consider this transition since considerably more time and memory will be required to take the transition into account. The second reason is the coupling mechanism not modeled in the simulation due to the use of smaller numbers of vias connecting the top and bottom stripline ground planes compared to the actual structures. This was done to save the computational time and memory. Therefore, the mismatch between CPW and strip line transition, via interconnection potentially causes the discrepancy between measurement and simulation. To save the computational time, each filter was simulated with 2 mils $\times 2$ mils subsections, and in "memory save mode" utilizing single-precision instead of double-precision variables. To obtain a better accuracy, the use of $1 \mathrm{mil} \times 1$ mil subsections and double-precision variables can be used even though more than five times of computing memory is required. 


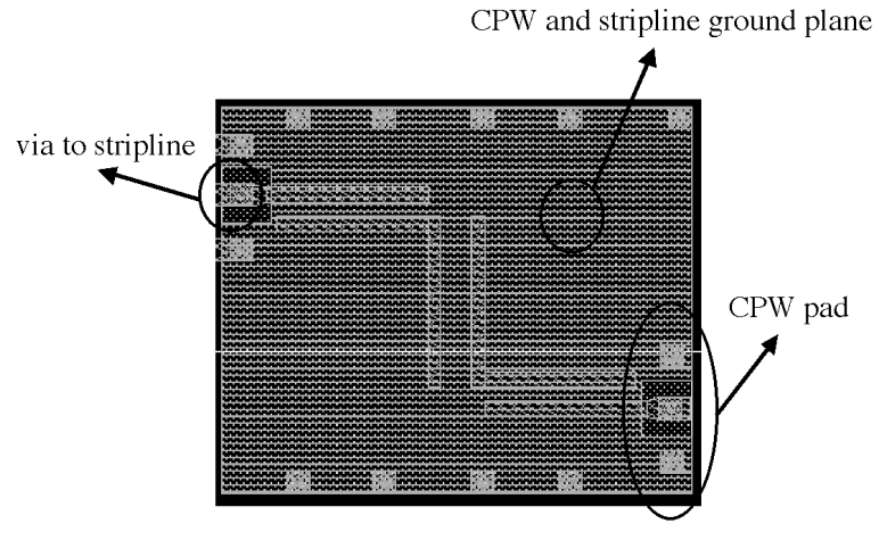

(a)

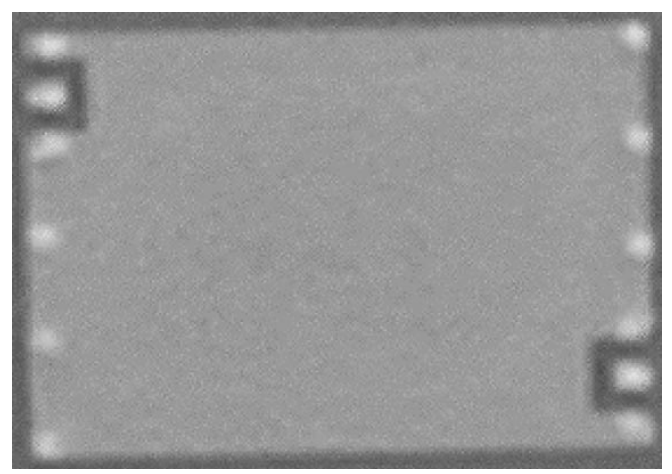

(b)

Fig. 15. (a) Top view of layout of the LTCC folded edge-coupled line filter with CPW pads and via to the strip line. (b) Photograph of implemented LTCC folded edge-coupled line filter.

Fig. 15(a) shows the layout of the designed coupled strip line filter with a folded edge structure, showing the coupled line trace embedded with 4 layers below the top layer. To allow on wafer characterization using coplanar probes, the input and output have to be on the same top layer which requires a good strip line to $\mathrm{CPW}$ transition and exhibits additional parasitic mechanisms. CPW pad for on wafer measurement along with via transition from strip line to $\mathrm{CPW}$ is also shown.

It has a compact size of $5.5 \mathrm{~mm} \times 3.8 \mathrm{~mm} \times 0.7 \mathrm{~mm}$ with the CPW pads and $3.8 \mathrm{~mm} \times 2.4 \mathrm{~mm} \times 0.7 \mathrm{~mm}$ without the measurement pads as shown in Fig. 15(b). An on-board integrated ceramic filter offers an alternative implementation to an on-chip active filtering, with trade-offs in terms of size, loss performance, power consumption and dynamic range. One of the advantages of this configuration is that it can be integrated on the substrate where the MMIC module is mounted without any assembly efforts. The LTCC process uses screen printing as well as low-loss stacked via processes and high conductivity metallization useful for high frequency applications. The substrate material is 3.7 mils thick, 951 stackable ceramic tape from Dupont and fabricated by National Semiconductor Corporation. The metallization of the buried layers is a $7 \mu \mathrm{m}$ thick silver alloy and the surface metallization is a $7 \mu \mathrm{m}$ wire-bondable electroplated gold. A familiar implementation of the distributed filter uses cascaded edge-connected quarter wavelength

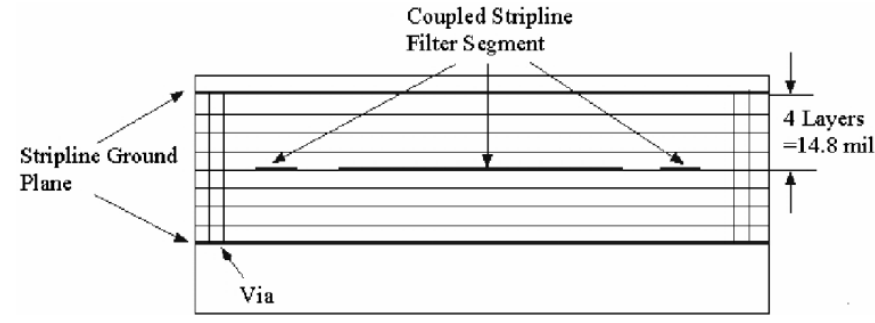

Fig. 16. Cross-sectional view of a folded edge-coupled line filter.

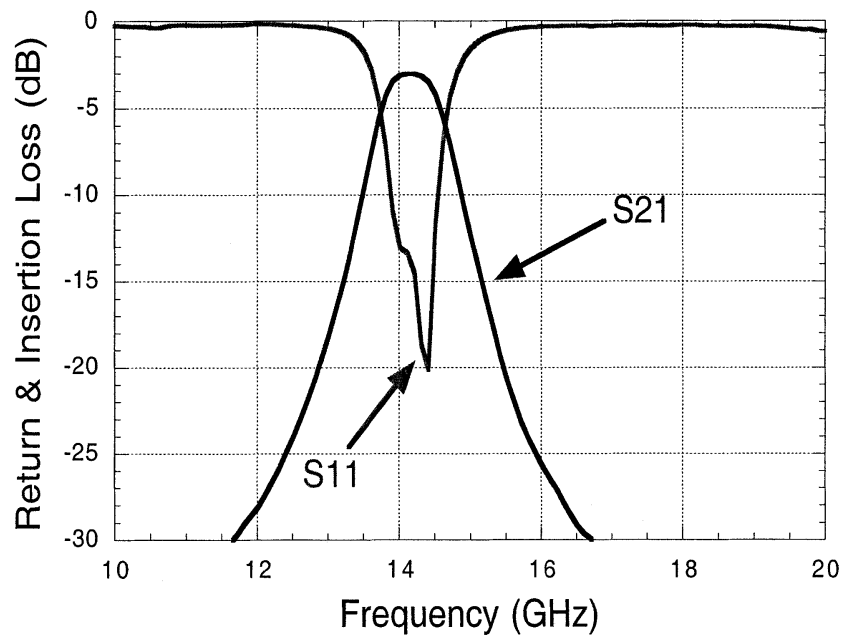

Fig. 17. Measurement result of Ku-band filter.

coupled-lines [10]. The conventional version of such a filter cascades the coupled line segments laterally on a single layer circuit board. Implementation of the filter in a strip line topology is desirable because it allows component placement on the surface layer to be in the same location as the filter in the $x-y$ direction. This would have been impossible if the filter were implemented in microstrip configuration. The two strip line ground planes are physically connected by vias.

The actual input and output are connected to the RF amplifier and driver amplifier, respectively, from an MMIC transmitter chip sets via wire-bonds. Fig. 16 depicts a cross-section of the LTCC tape stack-up, showing the eight layers used to create a strip line environment. This is a balanced strip line topology in which the coupled line segments are sandwiched by two ground planes at an equal distance of 14.8 mils (four tape layers). The first and third segments of the selected filter $\mathrm{E}$ have dimensions of coupled line length, width, and gap of 68,7 , and 6 mils, respectively. The second segment has dimensions of 80,6 , and 18 mils, respectively.

Fig. 17 shows the measured insertion loss and return loss of the filter $\mathrm{E}$ from frequency range of 10 to $20 \mathrm{GHz}$. This filter exhibits a maximum insertion loss of $3 \mathrm{~dB}$ from 14.0 to $14.5 \mathrm{GHz}$ with the corresponding return loss as high as $20 \mathrm{~dB}$ at $14.5 \mathrm{GHz}$. For a double conversion VSAT scheme, the IF frequency is at $1 \mathrm{GHz}$, the filter rejection at the image frequency of $12 \mathrm{GHz}$, and a $\mathrm{LO}$ frequency of $13 \mathrm{GHz}$ is about $28 \mathrm{~dB}, 20 \mathrm{~dB}$, respectively. An improved return loss is expected by adding a $50-\Omega$ strip line segment as an interface, connected by a tapered line segment to the input and the output of the filter to allow for a better impedance match. 


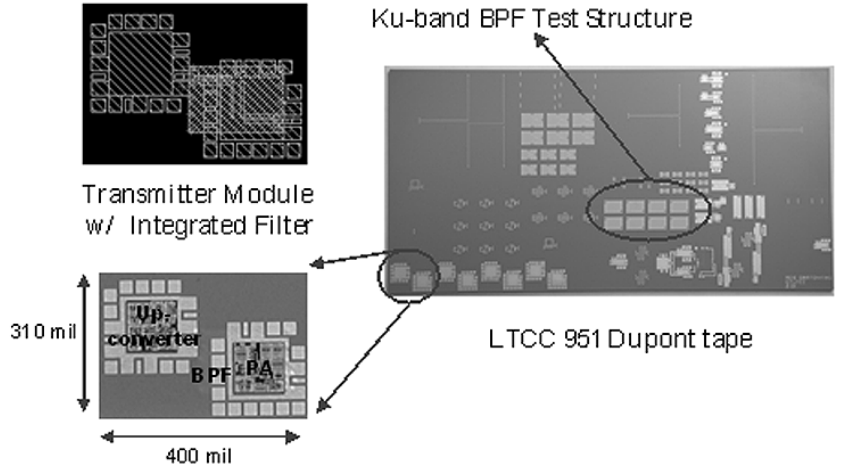

Fig. 18. Photograph of the implemented LTCC coupon and stripline filters.

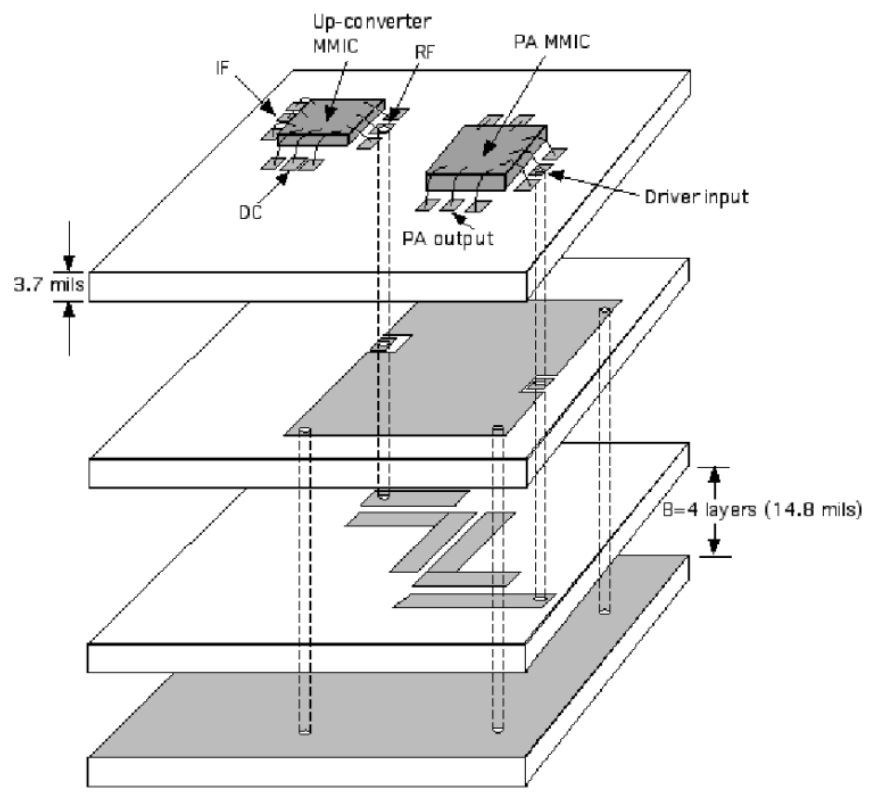

Fig. 19. Exploded view of transmitter MCM module with LTCC integrated filter.

\section{LTCC-BAsed Module Design AND Measured SYSTEM PERFORMANCE}

A photograph of a implemented LTCC 951 coupon including four different completed transmitter modules and eight different test structures for coupled strip line BPF is shown in Fig. 18. The module occupies a compact area of $400 \times 310 \times 35.2 \mathrm{mil}^{3}$. Fig. 19 depicts the three-dimensional exploded view of the LTCC module in which the MMIC chips were wire-bonded on the surface. The length of the wire-bond is approximately 40 mil. The estimated loss of 40 mil ball crescent bond wires incorporated in the module at $14 \mathrm{GHz}$ is $2 \mathrm{~dB}$ [11]. The filter ground planes were properly connected to the ground pads on the surface layers and are wire-bonded to the ground pads on the MMICs. The filter input was transitioned to the CPW line and wire-bonded to the output of the RF amplifier while the output transition of the CPW was wire-bonded to the input of the driver amplifier. Such a configuration where the filter is integrated between the mixer and the PA was chosen to eliminate the mixer harmonics and the linearity of the transmitter was improved. The transition to $\mathrm{CPW}$ also enables separate

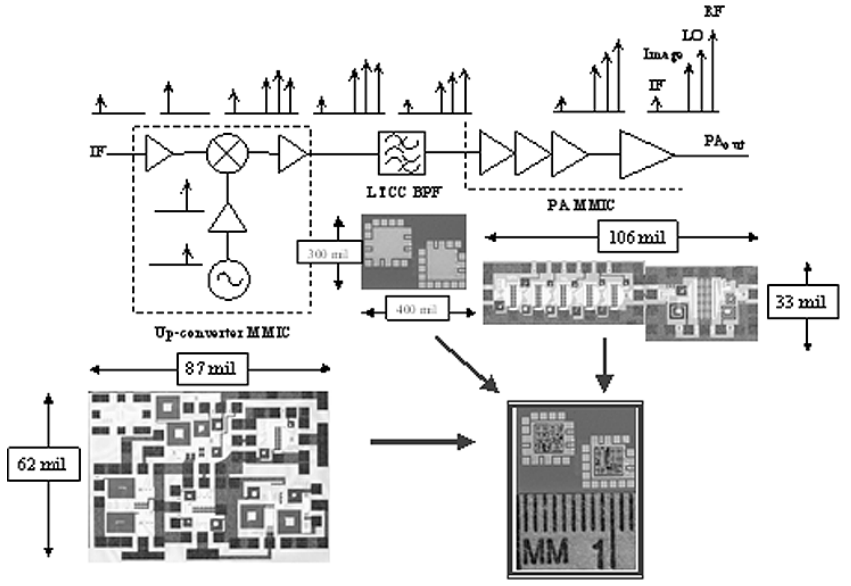

Fig. 20. Double conversion transmitter block diagram with output spectrum at each block.

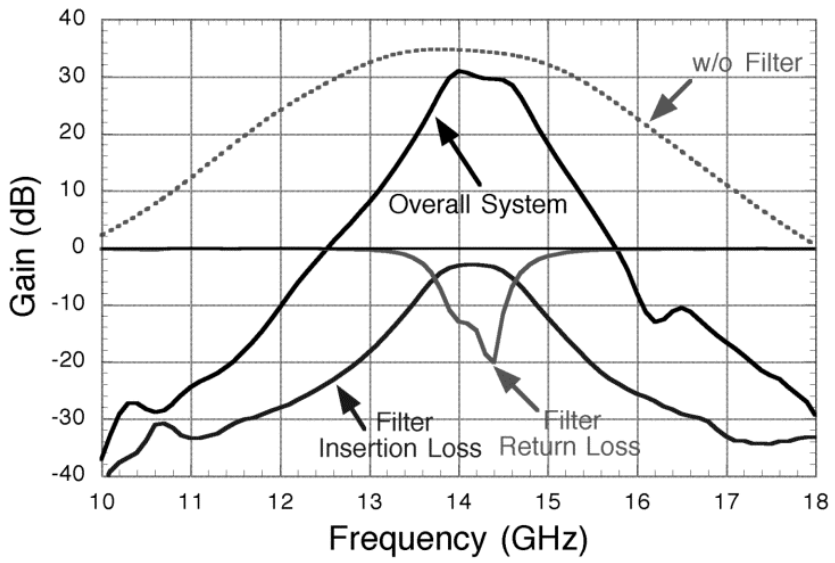

Fig. 21. Overall system gain performance with filter characteristics.

measurement of the filter. Fig. 20 shows the double conversion transmitter block diagram and output spectrum at the output of each block. The LO and image signal is attenuated after the BPF in the transmitter chain. Fig. 21 shows the measured overall system gain performance with filter characteristics. The entire transmitter chain exhibits a total conversion gain of $41 \mathrm{~dB}$ and output power of $26 \mathrm{dBm}$ incorporating the filter and wire-bond losses from $14 \mathrm{GHz}$ to $14.5 \mathrm{GHz}$ and $\mathrm{LO}$ rejection of $20 \mathrm{dBc}$ as well as image rejection of more than $40 \mathrm{dBc}$. Fig. 22 shows the output spectrum of up-converter for a data rate of $4 \mathrm{Mbps}$ and $16 \mathrm{Mbps}$, respectively. The operability for Ku-band application requires an output power of more than $24 \mathrm{dBm}$ [12]. Spectrum mask for Ku-band system [12] requires relative power spectrum levels of $-9,-16,-26 \mathrm{~dB}$ at $0.3 R, 0.35 R$, $0.5 R$ frequency offsets from the center frequency, where $R$ is defined as the bit rate entering the modulator. Measurement results of the chipset indicate that the output spectrum of the developed module fits well within the specified spectrum mask for the data rates of $64,128,192$, and up to $32 \mathrm{Mbps}$ as shown in Fig. 23, making it suitable for satellite outdoor units. ACPR analysis has been performed to estimate the power leakage between adjacent channels due to RF front-end nonlinearities. There are 6 channels within the $210 \mathrm{MHz}$ band as per direct broadcasting satellite (DBS) standard [12], hence 


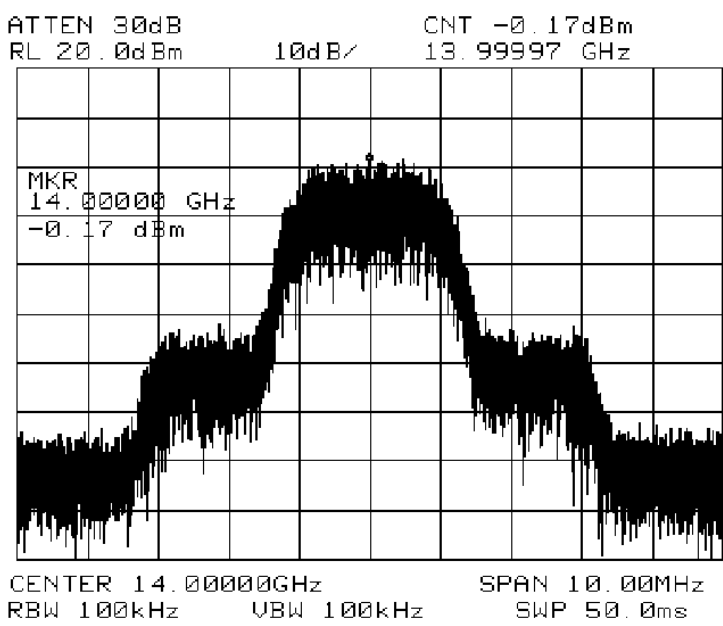

(a)

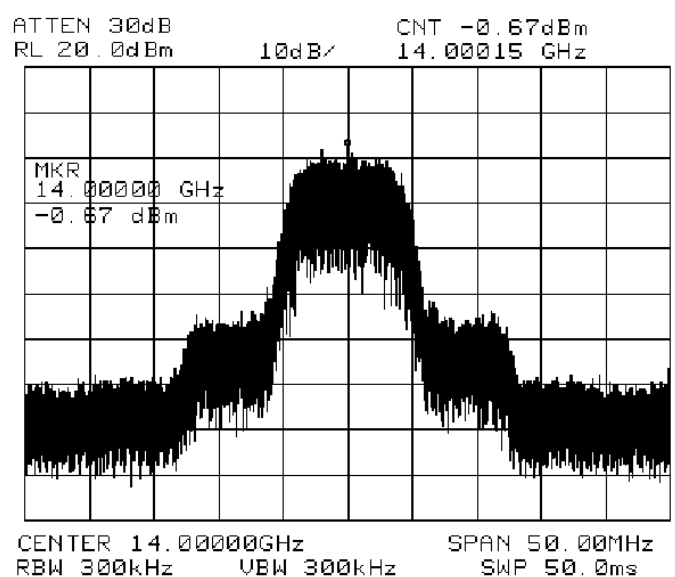

(b)

Fig. 22. Output power spectrum from up-converter with QPSK modulation (a) 4 Mbps QPSK modulation and (b) 16 Mbps QPSK modulation.

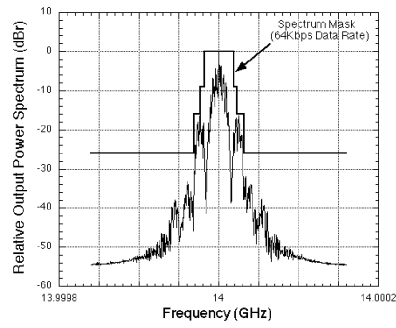

(a)

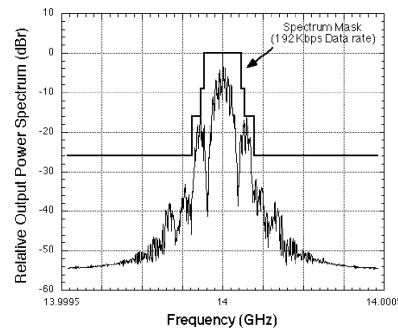

(c)

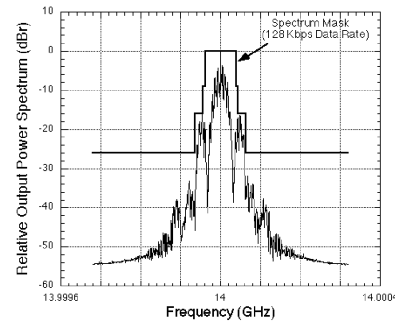

(b)

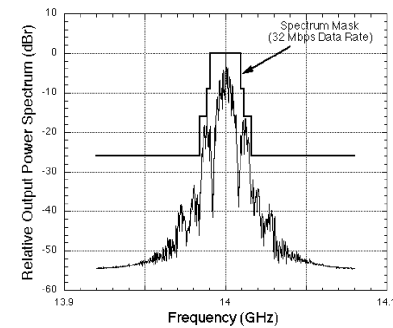

(d)
Fig. 23. Output power spectrum with the spectrum mask. (a) 64 Kbps, (b) 128 Kbps, (c) $192 \mathrm{Kbps}$, and (d) 32 Mbps.

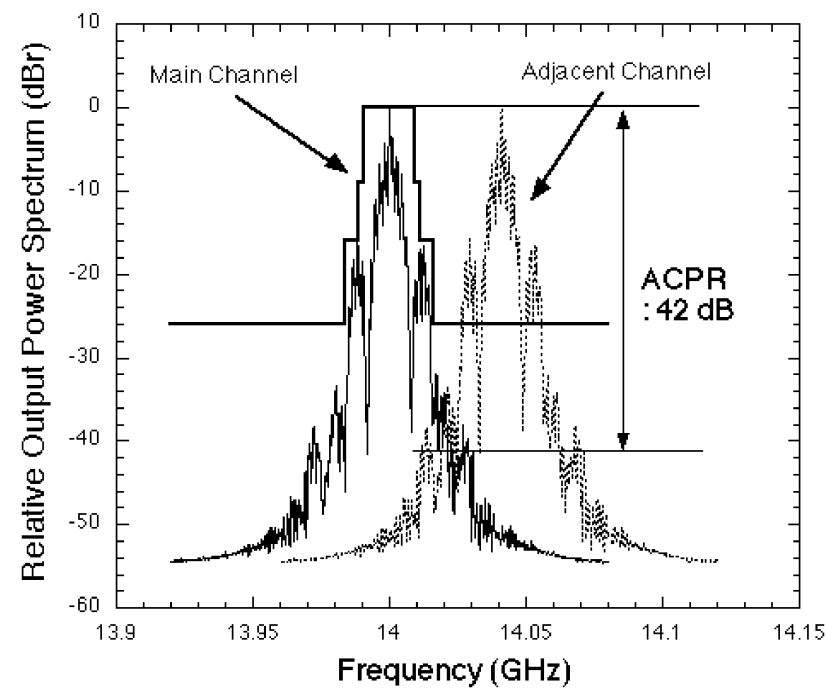

Fig. 24. ACPR with $32 \mathrm{Mbps}$ data rate.

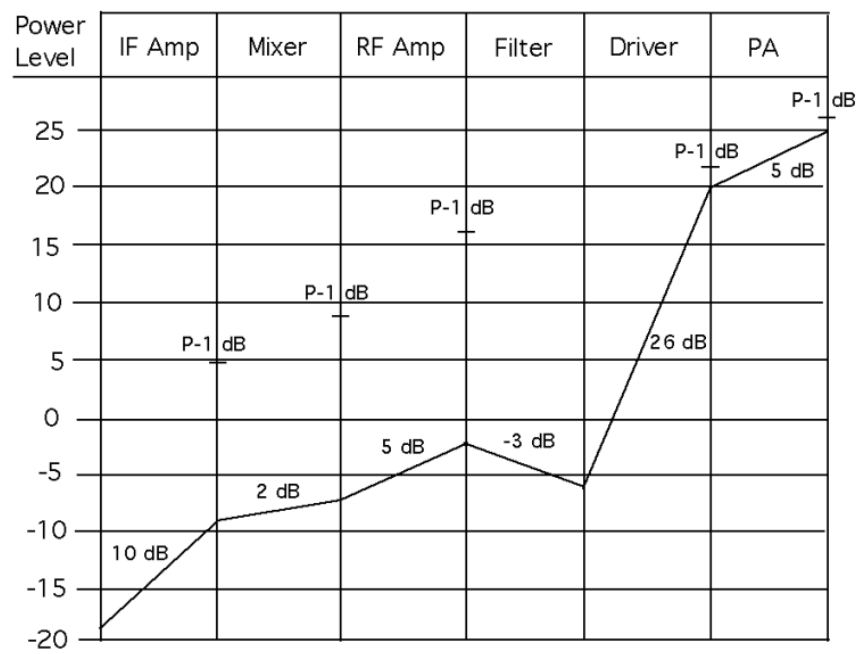

Fig. 25. Level diagram of the entire transmitter system with 1-dB compression point.

TABLE II

Transmitter Module System Performance at $14 \mathrm{GHz}$, With a LO (13 GHz) POWER OF $7 \mathrm{dBm}$ AND AN IF (1 GHz) POWER OF $-15 \mathrm{dBm}$

\begin{tabular}{c|c}
\hline Transmitter Module Performance & \\
\hline Up-conversion Gain $(\mathrm{dB})$ & 41 \\
\hline Output P1dB $(\mathrm{dBm})$ & 26 \\
\hline 3dB Bandwidth $(\mathrm{GHz})$ & 1.0 \\
\hline LO Rejection $(\mathrm{dB})$ & 13 \\
\hline Image Rejection $(\mathrm{dB})$ & 40 \\
\hline Data Rates $(\mathrm{Kbps})$ & Up to 32064 \\
\hline ACPR $(\mathrm{dB})$ & 42 \\
\hline
\end{tabular}

the adjacent channels are taken to be $41 \mathrm{MHz}$ apart from each other. Measured performance of chipset indicated an ACPR of $42 \mathrm{~dB}$ for the transmitter module with a data rate of $32 \mathrm{Mbps}$ as shown in Fig. 24. Fig. 25 shows the measured system level diagram and $1-\mathrm{dB}$ power compression performance of the 
entire transmitter chain based on the measurement of each transmitter blocks. Table II summarizes the overall system performance of transmitter module.

\section{CONCLUSION}

This paper has presented the first demonstration of a compact LTCC-based transmitter module with functional MMICs, which consist of VCO-mixer and PA implemented in a commercial GaAs MESFET technology. The up-converter MMIC demonstrated the conversion gain of $15 \mathrm{~dB}$ and IIP3 of $15 \mathrm{dBm}$. The PA MMIC exhibits the total gain of $31 \mathrm{~dB}$ and $1-\mathrm{dB}$ compressed output power of $26 \mathrm{dBm}$. The compact module was made possible by embedding the filter and thereby reducing the size of the area required by $40 \%$ compared to such a module implemented on a typical alumina substrate. The integrated strip line filter inserted between the mixer and the PA demonstrates a low insertion loss of $3.0 \mathrm{~dB}$ from 14 to 14.5 GHz. The transmitter module exhibits the total conversion gain of $41 \mathrm{~dB}$ and output power of $26 \mathrm{dBm}$ incorporating wire-bond and filter losses. Measured performance of the chipsets indicate that the output power spectral density at various data rates conforms to the spectral mask specification, thereby proving the applicability of the developed transmitter module in the $\mathrm{Ku}$-band satellite communication standard. This ultra-compact module is an attractive solution for low-cost $\mathrm{Ku}$-band satellite outdoor units. These approaches suggest the feasibility of building highly SOP integrated microwave and millimeter wave radio front-end development.

\section{ACKNOWLEDGMENT}

The authors would like to thank R. Brown and M. Ehlert, National Semiconductor, for fabricating the LTCC prototypes, as well as the Staff of Triquint Semiconductor, for MMIC fabrication.

\section{REFERENCES}

[1] J. V. Evans, "Proposed U.S. global satellite systems operating at Ka-band," in Proc. IEEE Aerosp. Conf., vol. 4, 1998, pp. 525-537.

[2] W. Simon, R. Kulke, A. Wien, M. Rittweger, L. Wolff, A. Girard, and J.-P. Bertinet, "Interconnects and transitions in multilayer LTCC multichip modules for $24 \mathrm{GHz}$ ISM-band applications," in Proc. 2000 IEEE MTT-S Int. Dig., vol. 2, Boston, MA, pp. 1047-1050.

[3] K. Fujii, Y. Hara, Y. Shibuya, T. Sakai, and Y. Takano, "Highly integrated T/R module for active phased array antennas," in Proc. 1998 IEEE RFIC Dig., Baltimore, MD, pp. 77-80.

[4] J. W. Gipprich, L. E. Dickens, and J. A. Faulkner, "Power amplifier yields $10 \mathrm{~W}$ over $8-14 \mathrm{GHz}$ using GaAs MMICs in an LTCC serial combiner/divider network," in Proc. 1993 IEEE MTT-S Int. Dig., vol. 3, Atlanta, GA, pp. 1369-1372.

[5] A. Sutono, J. Laskar, and W. R. Smith, "Development of three dimensional integrated bluetooth image reject filter," in Proc. IEEE MTT-S Int. Dig., vol. 1, Boston, MA, June 2000, pp. 339-342.

[6] J. A. Lester, M. Ahmadi, S. Peratoner, J. Hathaway, D. Garske, and P. D. Chow, "Low cost miniaturized EHF SATCOM transceiver featuring HEMT MMICs and LTCC multilayer packaging," in Proc. IEEE Microw. Millimeter-Wave Monolithic Circuits Symp. Dig., 1995, pp. 35-38.

[7] C.-H. Lee, S. Han, B. Matinpour, and J. Laskar, "A low phase noise X-band MMIC GaAs MESFET VCO,” IEEE Microwave Guided Wave Lett., vol. 10, pp. 325-327, Aug. 2000.

[8] EM Users Manual. Liverpool, NY: Sonnet Software Inc.
[9] M. T. Jones and J. T. Bolljahn, "Coupled strip transmission line filters and resonators," IRE Trans. Microwave Theory Tech., vol. MTT-4, pp. 75-81, Apr. 1956.

[10] S. B. Cohn, "Parallel coupled transmission-line-resonator filters," IRE Trans. Microwave Theory Tech., vol. MTT-6, pp. 223-231, Apr. 1958.

[11] A. Sutono, N. G. Cafaro, J. Laskar, and E. M. Tentzeris, "Experimental study and modeling of bond wire interconnects for microwave integrated circuits," in Proc. IEEE AP-S Conf., vol. 4, Salt Lake City, UT, Jan. 2000, pp. 2020-2023.

[12] INTELSAT Earth Station Standards (IESS) Document IESS-208, Tech. Rep., 2002.

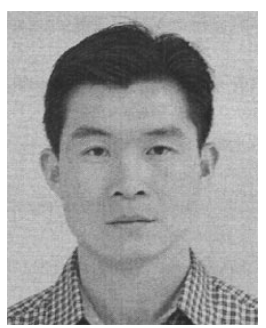

Chang-Ho Lee (M'01) received the B.S. and M.S degree in electrical engineering from Korea University, Seoul, Korea, in 1989 and 1991, respectively, and the M.S. and Ph.D. degrees in electrical and computer engineering from Georgia Institute of Technology, Atlanta, in 1999 and 2001, respectively.

He was a Research Engineer with DACOM Corporations, Korea, for three years. In 2000, he joined RF Solutions, Inc., Norcross, GA, where he is a Staff Engineer. His research interest includes satellite communication system simulation, design, and characterization of the transceiver MMICs in GaAs MESFET, pHEMT, and HBT processes, as well as LTCC-based multilayer multichip modules development for satellite and wireless communication applications. His current research is related to dual-band transceiver design for WLAN applications.

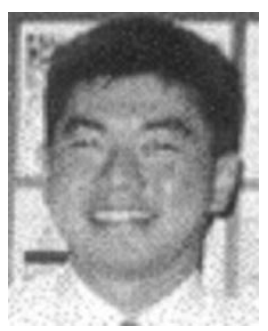

Albert Sutono received the Ph.D. degree in electrical engineering from the Georgia Institute of Technology, Atlanta, in 2001.

$\mathrm{He}$ has one U.S. patent pending and authored/co-authored over 40 peer-reviewed IEEE and international journal and conference publications in the area of ceramic and organic-based microwave and millimeter wave integrated modules, in addition to several invited talks. He held a position as a M(M)IC Design Engineer at RF Solutions, Norcross, GA, before joining Infinera, Inc., Sunnyvale, CA, in 2002. His current research is millimeter wave-photonic device modeling and characterization.

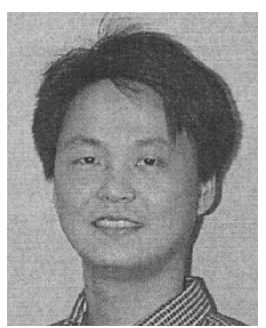

Sangwoo Han received the B.S. degree from Carnegie-Mellon University, Pittsburgh, PA, in 1992, the M.S. degree from the University of Pennsylvania, Philadelphia, in 1993, and the Ph.D. degree from the Georgia Institute of Technology, Atlanta, in 2000. His doctoral work includes development of MMIC-based mult-chip and single-chip transmitter modules for an optical sub-carrier multiplexing (OSCM) communication link.

He was responsible for the complete design and characterization of all MMIC chipsets, filters, and boards for these modules. In 1998, he joined RF Solutions, Inc., Norcross, GA, where he has developed GaAs MESFET $3.5 \mathrm{GHz}$ and UNII band power amplifiers for fixed wireless applications and GaAs HBT UNII band power amplifiers for wireless LAN applications. He has also developed a model for implementation of a W-CDMA RF sub-system focusing on optimization of system performance and cost. He has over seven years of experience in RF sub-system and MMIC designs. Prior to RF Solutions, he worked in the areas of RF sub-system simulations and RF transceiver designs at Electronics and Telecommunications Research Institute (ETRI), Korea, and Hewlett-Packard (HP). He has written and contributed to several Microwave Theory and Techniques (MTT) Journal publications and presented at many conferences on $\mathrm{RF} /$ optical communications and RF transceiver design. 


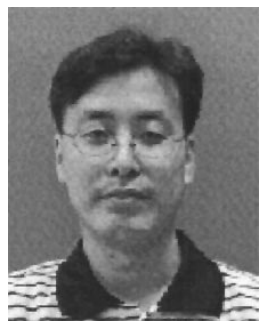

Kyutae Lim (M'99) was born in Seoul, Korea, in 1968. He received the B.S., M.S., and Ph.D. degrees from Hanyang University, Seoul, Korea, in 1991, 1993, and 1996, respectively.

From 1996 to 2000, he was with the Samsung Advance Institute of Technology, Kiheung, Korea, as a Technical Staff, Member where he was involved in developing mm-wave wireless LAN systems. From 1998 to 1999, he was with the Communication Research Laboratory, Tokyo, Japan, as a Research Fellow, where he developed planar antennas and packages for $60 \mathrm{GHz}$. In January 2000, he joined the Microwave Application Group, Georgia Institute of Technology, Atlanta, as a Research Engineer. He has authored and co-authored over 40 papers and holds two patents. His research interests include the development of the passive/active circuits for $\mathrm{RF} /$ wireless and high-speed opto-electronic applications. He also interested in the mixed signal system integration by the system-on-packaging concept.

Dr. Lim is a member of IEEE Microwave Theory, Component, Packaging, and Manufacturing Technology, and Technique and Antennas and Propagation Societies.

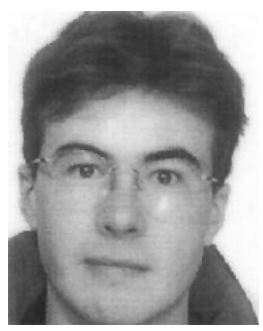

Stéphane Pinel was born in Toulouse, France, in 1974. He received the B.S. degree from Paul Sabatier University, Toulouse, France, in 1997 and the Ph.D. degree in microelectronics and microsystems from the Laboratoire d'Analyze et d'Architecture des Systemes, Centre National de la Recherche Scientifique, Toulouse, in 2000.

He has worked on a ultrathin chip stacking (UTCS) European Project for three year and is currently doing Post-Doctoral work at the Microwaves Applications Group, Georgia Institute of Technology, Atlanta. His research interests include advanced 3-D integration and packaging technologies, RF packaging and RF-MEMS, system-on-package for RF front-end module, and SOI RF circuit design.

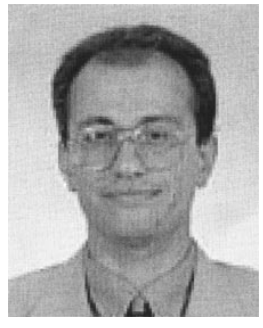

Emmanouil M. Tentzeris (M'99) received the Diploma degree in electrical engineering and computer science (with high honors) from the National Technical University, Athens, Greece, in 1992 and the M.S. and Ph.D. degrees in electrical engineering and computer science from the University of Michigan, Ann Arbor, in 1993 and 1998, respectively.

He joined the School of Electrical and Computer Engineering, Georgia Institute of Technology (Georgia Tech), Atlanta, in 1998 as an Assistant Professor, and he is currently the leader of the Novel Integration Techniques Subthrust, Broadband Hardware Access Thrust, Yamacraw Initiative, State of Georgia, and the Packaging Research Center Thrust Leader for RF/Wireless Packaging. At Georgia Tech, he has established academic programs in numerical electromagnetics, RF/wireless applications, packaging adaptive ultracompact antennas, and RF MEMS modeling: over 75 publications, three book chapters, and numerous invited papers.

Dr. Tentzeris received the National Science Foundation CAREER Award (2000), the Georgia Tech-ECE Outstanding Junior Faculty Award (2002), the International Hybrid Microelectronics and Packaging Society Best Paper Award (1997), and the Applied Computational Electromagnetics Society Best Paper Award (2001). He has been an Invited Professor at the Technical University of Muenchen, Munich, Germany, Summer 2002. He was the 1999 Technical Program Co-Chair of the 54th ARFTG Conference and he is currently a member of the Technical Program Committees of IEEE-IMS and IEEE-AP Symposiums. He is the Vice-Chair of IEEE-CPMT TC16 (RF Subcommittee).

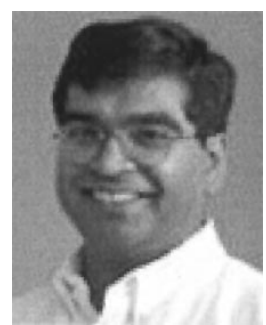

Joy Laskar (M'91) received the B.S. degree (with highest honors) in computer engineering from Clemson University, Clemson, SC, in 1985 and the M.S. and Ph.D. degrees in electrical engineering from the University of Illinois, Urbana-Champaign, in 1989 and 1991, respectively.

Prior to joining the Georgia Institute of Technology (Georgia Tech), Atlanta, in 1995, he held faculty positions at the University of Illinois and the University of Hawaii. At Georgia Tech, he is currently the Chair for the Electronic Design and Applications Technical Interest Group, the Director of Research for the state of Georgia's Yamacraw Initiative, and the NSF Packaging Research Center System Research Leader for RF and Wireless. His research has focused on high frequency IC design and their integration. At Georgia Tech, he heads a research group of 25 members with a focus on integration of high frequency electronics with optoelectronics and integration of mixed technologies for next generation wireless and optoelectronic systems. His research is supported by over 15 companies and numerous federal agencies including: DARPA, NASA and NSF. He has published over 100 papers, numerous invited talks and has 10 patents pending.

Dr. Laskar received the Army Research Office's Young Investigator Award in 1995, the National Science Foundation's CAREER Award in 1996, the NSF Packaging Research Center Faculty of the Year in 1997, the NSF Packaging Research Center Educator of the Year in 1998, the IEEE Rappaport Award (Best IEEE Electron Devices Society Journal Paper) in 1999, and the IEEE MTT IMS Best Paper award in 2000. He is a co-Organizer and Chair for the Advanced Heterostructure Workshop, serves on the IEEE Microwave Theory and Techniques Symposia Technical Program Committee, and is a member of the North American Manufacturing Initiative Roadmapping Committee, and is co-founder of a Broadband Wireless Company, RF Solutions. 